\title{
tic\&société
}

Vol. 12, $\mathrm{N}^{\circ} 1$ | 1er semestre 2018

Communs numériques et communs de la connaissance

\section{Communs numériques et communs de la connaissance}

Introduction

Hervé LE CROSNIER

(2) OpenEdition

Journals

Édition électronique

URL : http://journals.openedition.org/ticetsociete/2348

DOI : 10.4000/ticetsociete.2348

Éditeur

Association ARTIC

Édition imprimée

Pagination : 1-12

Référence électronique

Hervé LE CROSNIER, «Communs numériques et communs de la connaissance », tic\&société [En

ligne], Vol. 12, № 1 | 1er semestre 2018, mis en ligne le 31 mai 2018, consulté le 11 octobre 2020

URL : http://journals.openedition.org/ticetsociete/2348; DOI : https://doi.org/10.4000/ticetsociete. 2348 
tic\&société - 12(01), 2018

\title{
Communs numériques et communs de la connaissance
}

\author{
Introduction
}

\section{Hervé LE CROSNIER}

II est toujours difficile de définir les communs. Les activistes et les théoriciens qui se réfèrent à ce concept mettent en avant de nombreuses pratiques collectives en les considérant comme des communs. Cependant, pour les acteurs de ces pratiques, le concept est souvent ignoré, les spécificités de chaque action proposée l'emportant sur toute généralisation. Le film de Cyril Dion et Mélanie Laurent Demain ${ }^{1}$ présente des expériences de transition menées dans dix pays. On peut considérer que toutes les initiatives présentées s'intègrent dans la théorie des communs... et pourtant le terme n'est jamais prononcé dans le film. C'est le rôle de la théorie de forger des concepts pour donner à voir des convergences et des régularités parmi la diversité de la nature ou des relations sociales. Ainsi en est-il de la théorie des communs : nul besoin de s'en réclamer pour y participer, comme l'affirmait M. Jourdain, faisant de la prose sans le savoir; et, dans le même temps, il nous faut reconnaître la nécessité d'un concept unificateur qui permette aux acteurs sociaux concernés de se connaître mutuellement et de renforcer leur praxis (pratique et théorie de leurs actions).

Les communs ont rencontré le numérique. Les «Pères fondateurs " de l'Internet, les ingénieurs qui ont conçu ce réseau, avaient une ambition majeure : créer un réseau solide, ouvert, coopératif et garantissant l'horizontalité (échanges entre pairs) et la liberté d'expression. Sans utiliser le terme de communs lors de la mise en place des protocoles, par le choix d'une normalisation réalisée par les ingénieurs concernés euxmêmes, par la mise en avant de pratiques à la fois collectives et indépendantes (dont le motto "rough consensus and running code » est un des symboles), l'Internet s'est constitué

\footnotetext{
${ }^{1}$ Voir : https://www.demain-lefilm.com/.
} 
comme un gigantesque commun mondial. Une ressource partagée, mais également des formes de régulation décidées par les acteurs eux-mêmes, des règles de droit émanant de la communauté des développeurs et des usagers (au moins au début) du droit que l'on retrouve inscrit dans le code informatique lui-même ("code is law », disait Lawrence Lessig). Comme tout commun, l'Internet est fragile devant les formes d'enclosure, de réduction des usages communs. Pour Internet, celles-ci prennent aujourd'hui la forme de la concentration capitalistique des plateformes géantes autant que les menaces de cyberguerre ou de contrôle de la vie privée par des entités privées comme étatiques. En face de ces tentatives, on voit également se développer l'organisation d'une résistance à l'enclosure, une défense des communs du numérique.

L'existence de l'Internet a profondément marqué la théorie des communs, lui redonnant un nouveau souffle pour penser comme des communs des espaces immatériels et de grande dimension. Premier commun à se construire simultanément au réseau dans le monde numérique, le mouvement des logiciels libres a marqué la théorie par l'élaboration de règles de droit émanant de la communauté des développeurs, la fameuse licence "GNU-GPL », ou les autres « licences libres ». Car les communs ont à voir avec le droit, notamment en distinguant l'usage de la propriété, en créant un "faisceau de droits" selon l'expression du juriste américain du début du $X X^{\mathrm{e}}$ siècle John R. Commons (Orsi, 2013). Le " hack juridique » des licences a inspiré de nombreux mouvements parmi les producteurs de culture et de connaissance. Le premier d'entre eux est le mouvement de l'accès libre aux publications scientifiques. Le réseau permet de diffuser rapidement et pour un coût marginal proche de zéro les travaux réalisés dans les universités, et le droit d'auteur assure à l'auteur la " propriété » de son manuscrit, qu'il peut donc diffuser dans des archives ouvertes. II s'agit là d'une volonté de poursuivre le " communalisme » scientifique qui avait été la marque de la science de l'après-guerre, décrite notamment par le sociologue des sciences Robert K. Merton. Un communalisme que la valorisation des brevets dans les universités ou l'extension du brevetable au vivant et à la connaissance (algorithmes ou méthodes) avaient réduit considérablement à partir du début des années 1980 et que le mouvement pour l'accès libre aux publications scientifiques tente depuis 1991 de remettre au goût 
Communs numériques et communs de la connaissance.

Introduction

du jour, tout comme la création par Paul Ginsparg du dépôt arXiv $^{2}$. Suivant ces deux modèles issus des logiciels libres et des publications scientifiques, Lawrence Lessig a créé, au début des années 2000, les licences Creative Commons en adaptant le processus d'auto-gouvernement aux créateurs d'œuvres culturelles (textes, images, musique, vidéo...).

À l'hétérogénéité des pratiques concernées, ajoutons les deux grands angles de vue portés par les théoriciens. D'une part, dans la filiation d'Elinor Ostrom, qui a obtenu en 2009 le Prix de la banque de Suède, appelé médiatiquement "Nobel d'économie ", pour ses travaux sur les communs, on trouve des travaux qui développent une approche institutionnelle: comment les acteurs des communs trouvent-ils des arrangements permettant de gérer collectivement, en indépendance par rapport au marché comme à l'État, des ressources partagées ? D'autre part, à la suite de Michael Hart et Toni Negri, puis en France de Pierre Dardot et Christian Laval, on observe une approche du commun comme objet et objectif d'une action politique instituante.

C'est dans ce paysage évolutif, bourgeonnant et inventif, à la fois conscient de ses actions et parfois imperméable à la conceptualisation, que nous avons recueilli les articles présentés dans ce numéro de la revue tic\&société. Nous les avons regroupés en quatre thèmes.

\section{Commun(s) et pratiques culturelles}

Les pratiques culturelles forment le premier thème abordé. La définition même de la culture étant autant disputée que celle des communs, les trois articles sélectionnés offrent une pluralité de points de vue. Valérian Guillier s'est penché sur la notion de commun quand elle s'applique à la culture. A priori, la production culturelle est avant tout une question privée. Le droit d'auteur est ainsi un "droit de la personne ». Pourtant, l'ensemble culturel, la production des "biens symboliques", peut relever de la notion de commun, mais cela demande d'interroger certains fondamentaux de la production et de la diffusion de la culture. Au-delà des œuvres, la culture libre, comme le revendique Lawrence Lessig (2004), est un

${ }^{2}$ Voir : https://fr.wikipedia.org/wiki/ArXiv. 
processus de création ouverte, intégrant des œuvres du passé et autorisant le remixage des œuvres proposées. Plus que «culture » et "art », termes très connotés, l'auteur préfère parler de "biens symboliques", dont l'échange, ou la réécriture collective, est d'emblée organisé par les producteurs, notamment au travers des licences d'usage, car le commun culturel n'émerge pas de la simple capacité à reprendre les œuvres, qui est à la base du domaine public, mais dans la volonté de communautés de gérer la mise à disposition et le partage de biens symboliques. S'appuyant sur les travaux de Dardot et Laval, il s'agit de considérer les productions culturelles comme une action politique instituante, " comme un mouvement collectif et une ambition politique qui se nourrissent mutuellement $»$.

Lisiane Lomazzi et Marc Ménard se sont penchés sur l'économie politique de la culture à la lueur des communs, dans l'acception socioéconomique d'Elinor Ostrom, ou du commun dans la théorisation sociopolitique de Dardot et Laval. Dans tous les cas, il s'agit de passer d'une catégorisation des choses (biens) vers une définition des processus, comme arrangements institutionnels dans l'école de Bloomington d'Ostrom, ou comme dynamique instituante dans celle de Toni Negri ou de Dardot et Laval, malgré leurs différences. Le droit ne peut être considéré comme la représentation d'un " ordre naturel des choses ॥, mais comme un phénomène social, enregistrant simultanément des rapports de force économiques et des volontés collectives. II en va ainsi du droit de la " propriété intellectuelle », qu'il nous faut considérer en regard de la notion essentielle de faisceau de droits, en le rendant capable d'inclure les " droits " des usagers de la connaissance. À l'image de ce qu'a proposé la Commission Rodotà ${ }^{3}$ en 2007 en Italie, il apparaît fécond d'inscrire en droit les communs sous

\footnotetext{
${ }^{3}$ La Commission Rodotà a été mise en place en 2007 en Italie pour proposer une modification de la notion de propriété publique dans le Code civil. Les propositions rendues en 2008 ont été repoussées par le nouveau gouvernement italien. "Leur grande originalité était d'envisager une nouvelle catégorie de biens, différente des biens publics : celle des biens communs, dont la titularité, le contrôle, la possession, la gestion relèveraient de la collectivité dans son ensemble et non d'une structure administrative telle que l'État, et dont la législation devrait reposer sur certains principes fondamentaux et sur l'idée qu'ils constituent des biens par définition indisponibles, parce qu'ils sont indispensables à la satisfaction des besoins primaires de tous et de chacun " (Lucarelli, 2012, p. 2).
} 
Communs numériques et communs de la connaissance.

Introduction

le régime de l'inappropriable et de l'indisponible. Pour Lomazzi et Ménard, " il s'agirait donc d'opérer un rapprochement entre la réflexion de la socioéconomie des communs qui consiste à repenser la propriété dans le domaine du droit et celle, plus macrosociale, de la sociopolitique du commun ». Or une telle proposition est profondément affectée par l'irruption du numérique, qui permet des formes d'institutionnalisation directement inscrites dans le code informatique, ce qui crée un nouvel arrangement institutionnel, dont il importe de défendre la capacité d'action pour construire du commun, contre la conception d'un code (informatique ou juridique) limitant les usages et les échanges.

Dans le même esprit d'interrogation sur l'émergence d'une culture en commun(s), Maud Pelissier revient sur une lecture circonstanciée des travaux fondateurs de Lawrence Lessig, Yochaï Benkler et James Boyle, trois auteurs parmi celles et ceux qui ont bousculé les conceptions marchandes de la culture pour en appréhender les fondements juridiques d'un partage des savoirs. Un partage rendu d'autant plus possible et nécessaire que se développaient les réseaux numériques. Pour cela, il convient de revenir sur les conceptions du «droit d'auteur » et sur l'importance de disposer d'un domaine public rendu actif et mobilisable. Alors que beaucoup, aujourd'hui, considèrent ce droit comme un moyen d'imposer une « volonté de l'auteur sur le suivi de ses travaux", tous les auteurs précités rappellent qu'il s'agit au contraire d'un droit d'équilibre entre les intérêts de l'auteur (qui vont lui permettre de produire et de développer ses travaux) et celui de la société (dans les capacités à s'emparer des « œuvres utiles » comme le signalait dès 1710 le Statut d'Anne ${ }^{4}$ ). L'environnement numérique a de facto remis en cause l'effectivité du droit d'auteur en permettant aux individus, et non plus aux seules structures éditoriales, de diffuser et partager des œuvres. La stratégie des DRM (Digital Rights Management Systems) se révèle à ce titre être une forme de pénalité imposée sur les usagers/acheteurs en

\footnotetext{
${ }^{4}$ Édicté en 1710 en Angleterre, le Statut d'Anne est considéré comme la première loi traitant du copyright, même si la tradition française fait remonter le " droit d'auteur " à Beaumarchais, plus tard dans le siècle. En accordant le bénéfice d'un monopole sur son œuvre à l'auteur et non à l'éditeur (stationery), le Statut d'Anne veut développer un encouragement à l'éducation, en permettant aux hommes éclairés d'écrire des œuvres utiles à la société.

(Voir : https://en.wikipedia.org/wiki/Statute of Anne, consulté le 30 avril 2017.)
} 
limitant des droits évidents dans les modèles précédents (copie privée, prêt des œuvres, usages collectifs, notamment en bibliothèques, dans la recherche scientifique et l'éducation...). Or le numérique fait aussi surgir de nouvelles formes de communs culturels, de productions destinées dès leur conception à être partagées et ouvertes. Les exemples de Wikipédia, de Wattpad ou de la plateforme de musique Jamendo servent de support à une réflexion sur le partage, en complément de l'utilisation des licences d'usage telles les Creative Commons. L'irruption des amateurs dans le champ de la production culturelle diffusée permet d'imaginer la construction d'une forme de culture populaire inédite... en commun(s).

\section{Wikipédia : une gouvernance sous la loupe des communs}

Wikipédia est considéré, à juste titre, comme le plus grand des communs de la connaissance. Une encyclopédie écrite par ses lecteurs, proposant pas loin de trois cents langues et des millions d'articles, couvrant tous les sujets... Mais le succès et l'extension confrontent Wikipédia aux contraintes habituelles des grandes structures, avec des formes de bureaucratisation (quand la lettre des règles prend le pas sur leur esprit) et de retrait des intervenants, laissant aux administrateurs et autres experts de l'encyclopédie le soin de gérer l'ensemble. Les deux articles proposés, qui reconnaissent le caractère extraordinaire de l'encyclopédie collaborative et ouverte, portent leur regard sur des questions de gouvernance et rapportent des difficultés dans la mobilisation de toutes et tous, au profit de celles et ceux qui maîtrisent les règles et les tactiques de la gouvernance interne, avec des effets secondaires non négligeables sur la couverture de l'encyclopédie et sur la capacité à y inscrire des réflexions contemporaines qui n'auraient pas encore été validées par les médias.

Maude Gauthier et Kim Sawchuk se sont penchées sur le fonctionnement de Wikipédia à partir d'une expérience d'inscription dans l'encyclopédie de travaux portant sur le vieillissement, notamment des femmes. Les entrées de l'encyclopédie sont considérées comme des «objetsfrontières ", lieux d'échange autour duquel des approches 
Communs numériques et communs de la connaissance.

Introduction

différentes des savoirs considérés trouvent des espaces de négociation. Cependant, les auteures s'interrogent sur les formes de bureaucratie procédurale qui peuvent s'installer dans une telle encyclopédie ouverte, limitant l'apport de sujets controversés ou marginaux, ou abordés sous des angles différents. Alors que la question du vieillissement est dans l'encyclopédie rapportée à la médicalisation sous la forme de la gérontologie, les questions sociales sont évacuées. Les auteures soulignent la prééminence des «normes de rédaction » sur la qualité et l'intérêt du contenu. Les nouveaux venus doivent connaître une série de pratiques langagières et de citation qui ouvrent la porte à un rejet de la nouveauté et à un pouvoir disproportionné des "experts » de l'encyclopédie sur l'expertise du sujet traité. La question des communs ne pourra pas éviter de penser la bureaucratisation, phénomène inéluctable des grandes structures.

Cet enjeu de l'ouverture de la gouvernance se trouve également dans l'article de Gilles Sahut sur Wikipédia. II s'agit pour lui d'évaluer les modes et les pratiques d'élaboration des règles de l'encyclopédie. Les règles adoptées constituent un moyen de protéger l'encyclopédie des destructions et d'une utilisation/manipulation commerciale ou partisane. Toutefois, il convient de considérer la façon dont ces règles sont construites, modifiées et appliquées, au delà de la notion de transparence. La prééminence de quelques acteurs est un marqueur de l'encyclopédie (on considère que $5 \%$ des rédacteurs ont écrit $90 \%$ des articles, et moins de $1 \%$ ont participé aux modifications). L'élaboration des règles ellesmêmes est conduite suivant le même processus d'ouverture. Malgré cela, "des études montrent l'émergence d'une élite wikipédienne qui exerce une forte influence sur les prises de décision communautaires ». Le travail de Gilles Sahut porte sur l'historique des modifications de deux textes réglementaires: Citez vos sources et Vérifiabilité. La surreprésentation des "administrateurs » dans l'élaboration de ces règles y est flagrante.

Cet écueil des pratiques politiques qui existe dans la société en général est aussi sensible dans les communautés de communs, d'où la nécessité d'élaborer, à côté des arrangements institutionnels ostromiens (qui faisaient dire à Vincent Ostrom que les communs sont une manière de faire 
fonctionner des institutions avec des acteurs imparfaits), une éthique ou une philosophie des communs qui mérite de porter son attention sur la capacité d' " accueil » des communs envers les nouveaux venus et de leur offrir les moyens d'apprendre autant que de modifier les règles.

\section{Sciences participatives}

Comme souligné plus haut, l'organisation de l'accès libre aux publications scientifiques est un des premiers communs de la connaissance permis par l'extension du réseau Internet. Cela dit, au-delà, la numérisation du monde ouvre la porte à une conception de la science plus participative, dans laquelle les usagers, les makers, les diffuseurs et les médiateurs du savoir auraient leur mot à dire sur l'élaboration scientifique (mise en avant de thèmes qui ne correspondent pas forcément aux intérêts de l'innovation portée par les partenariats de recherche public-privé ; diffusion du savoir auprès des communautés et des acteurs concernés...). Deux expériences de mise en commun de pratiques scientifiques et techniques sont au sommaire.

Claire Brossaud nous présente Coexiscience, une structure hybride (coopérative, tiers-lieu, initiative de recherche) créée dans la région lyonnaise en France pour agir collectivement, entre chercheurs et avec les usagers et les innovateurs, de façon à transformer la science en commun. Dans l'acception traditionnelle de la science, par exemple telle qu'elle est définie par le sociologue des sciences Robert K. Merton, celle-ci est d'emblée considérée comme un commun, une pratique collective et ouverte, soumise à la critique constructive des autres chercheurs. Or l'article commence par montrer les diverses enclosures qui se portent sur l'activité scientifique, et notamment sur la diffusion de la science vers le public. Entre le dépôt de brevets, les prix prohibitifs des revues et les difficultés à trouver des financements de recherche en dehors des partenariats public/privés, il s'agit, pour résister à cette privatisation de la science, d'ouvrir un outil (structure, action, réseau) pour développer les sciences participatives et les voies nouvelles d'exploration de la nature et de la relation science/société. En tissant des relations entre les chercheurs institutionnels et les divers mouvements d'une science et d'une 
Communs numériques et communs de la connaissance. Introduction

technique prise en charge "en communs", comme les hackerspaces, les makers ou le succès de publications ouvertes, Coexiscience entend ouvrir de nouvelles perspectives. II convient notamment de transformer l'utilisateur de la science en coproducteur de savoir et en acteur de la diffusion des réflexions scientifiques : "Faire de la science un objet appropriable par le plus grand nombre représente aujourd'hui un défi sociétal ».

Peter Hanappe s'est interrogé, pour sa part, sur le rôle et les limites des licences d'usage à partir de la logique de construction d'un robot adapté aux « microfermes », c'est-à-dire de nouvelles formes d'agriculture liant petits espaces (notamment urbains) et usage des techniques numériques et robotiques. Une agriculture "numérique " se développe pour associer précision et respect du sol et des pratiques culturales. Fabriquer du matériel "libre " suffit-il à garantir la pérennité d'un commun du nouveau matériel agricole adapté à ces usages ? En réalité, ce sont les pratiques des agriculteurs usagers qui déterminent la place de tels robots et doivent pouvoir influer sur leur conception ou leur " réingeneering ". Dès lors, la mise en place de "labels ", forme d'inscription intellectuelle permettant de guider et d'encadrer, apparaît comme une voie prometteuse pour les communautés de pratiques. À l'image de ceux qui ont permis l'éclosion et finalement le succès de l'agriculture biologique, de tels labels pourraient protéger réellement le commun associant innovations techniques et pratiques paysannes. Alors que la captation de données agricoles sert aujourd'hui principalement les fabricants industriels de matériel, un commun de la fabrication libre (c'est-à-dire disposant de plans ouverts et reproductibles) ne peut se construire en dehors de l'accord et de la participation des agriculteurs concernés. Les communs de la connaissance ne peuvent se limiter à l'immatériel, et cet article nous rappelle l'importance des règles portant sur la partie intangible, même quand, au final, il s'agit de pratiques directement matérielles et même essentielles à la vie comme l'agriculture. 


\section{Action publique}

La question des communs, par laquelle les acteurs participent directement aux formes de gouvernementalité, interroge nécessairement les pouvoirs publics les plus proches des usagers que sont les municipalités et, plus globalement, les collectivités territoriales. Le mouvement des données ouvertes a longtemps été considéré comme un premier pas nécessaire pour construire des capacités d'action, de mobilisation et, plus largement, d'empowerment des citoyens. La logique d'ouverture des données collectées par les puissances publiques ne va pourtant pas de soi. Une donnée n'est jamais "neutre ». Elle a été collectée pour une raison précise, qui influence son contenu. La mise à disposition impose donc un travail de la donnée, qui lui-même est organisé par les collectivités en fonction de ce qu'elles imaginent être leur futur usager, au risque de privilégier les offreurs de services sur la capacité d'action autonome et coopérative des habitants.

Valérie Laroche, Marie-France Peyrelong et Philippe Beaune se sont penchés sur la logique de l'open data des collectivités territoriales, ou plus précisément des conditions qui permettent de transformer des données publiques en commun. Pour des raisons légales comme pour marquer la transparence, les collectivités territoriales tendent à rendre accessibles les données produites pour leur gestion. Or suffit-il de mettre des données à disposition pour en faire un commun ? Les données sont souvent un mythe, une abstraction qui viserait à faire croire que des informations pourraient être directement réutilisables en dehors des conditions pour lesquelles elles ont été produites. Les collectivités doivent « travailler » les données pour les rendre accessibles. Comment associer les futurs utilisateurs à ce travail de la donnée, assurer une gouvernance ouverte de la mise à disposition? Quelles licences d'usage permettent que les données ouvertes soient également libres pour toute utilisation, y compris quand il s'agit de réutilisations commerciales, dans lesquelles les données numériques sont considérées comme des infrastructures publiques? Les données ouvertes sont souvent considérées comme un service rendu aux usagers (éventuellement par l'intermédiaire de sociétés de service). Comment imaginer une gouvernance des données qui permette l'empowerment des citoyens, tant dans le choix des données à collecter, que dans leur usage et dans la 
Communs numériques et communs de la connaissance.

Introduction

définition des actions que peuvent mener les collectivités? L'exemple de la ville de Bologne est à ce titre significatif : elle associe des chercheurs, des élus et la population au travers de Chartes des communs urbains. Suivant David Bollier ${ }^{5}$, les auteurs estiment qu'il n'y a du commun que lorsqu'il y a une communauté ou un groupe qui va gérer les ressources, ici les données. Ils appellent donc à une nouvelle étape du mouvement des données ouvertes, associant les futurs usagers dès la conception et les usages des portails numériques territoriaux, notamment en leur permettant d'agir sur la structure et le contenu de tels portails.

Cet ensemble d'articles est loin d'épuiser les multiples problématiques des communs numériques ou de la connaissance, mais un large champ est ainsi couvert et peut initier de nouvelles réflexions, car, finalement, la connaissance, ce qui est de l'ordre de l'immatériel, a toujours une finalité portant sur des conditions matérielles d'existence. En considérant la connaissance comme un commun, qui peut de surcroît s'appuyer sur l'infrastructure numérique, on soulève de multiples questions sur l'organisation de notre vie en commun(s), pour faire face aux conditions concrètes et immédiates de l'existence, autant que pour agir sur les cadres globaux, notamment la question du changement climatique, des inégalités et des risques de guerre qui plombent l'avenir du $\mathrm{XXI}$ siècle.

\footnotetext{
${ }^{5}$ David Bollier, La renaissance des communs : pour une société de coopération et de partage, ECLM, 2014. http://www.eclm.fr/ouvrage-364.html
} 


\section{Hervé LE CROSNIER}

\section{Références}

Dardot, P. et Laval, C. (2014). Commun : essai sur la révolution au $X X I^{\ominus}$ siècle. Paris, France : La Découverte.

Lessig, L, (2004). Free Culture. New York, NY: The Penguin Press. Repéré à www.free-culture.cc/freeculture.pdf

Lucarelli, A. (2012) Au sein de la Constitution et au-delà de la Constitution. À la recherche de nouvelles dimensions de droit public : participation et biens communs. Séminaire du public au commun. Repéré à

http://seminaire.samizdat.net/spip.php?article271

Orsi, F. (2013). Elinor Ostrom et les faisceaux de droits: l'ouverture d'un nouvel espace pour penser la propriété commune. Revue de la Régulation, 14 . Repéré à http://regulation.revues.org/10471 